\title{
Theoretical analysis of Marangoni instability of an evaporating droplet by energy method
}

\author{
Vai-Meng Ha, Chun-Liang Lai * \\ Department of Mechanical Engineering, National Taiwan University, Taipei 106, Taiwan, ROC
}

Received 23 July 2003; received in revised form 15 March 2004

Available online 6 May 2004

\begin{abstract}
Evaporation of a droplet is a process of heat and mass transports. When the droplet evaporates, heat is removed from the surface and the surface temperature is reduced thereby, resulting in a temperature gradient normal to the free surface. Such a situation is similar to that with a liquid layer heated from below. Thus, Marangoni instability as described by Pearson [J. Fluid Mech. 4 (1958) 489] may probably exist in an evaporating droplet, which is a very important and interesting phenomenon both from the academic and application points of view. In the present work, the energy method, which is appropriate for unsteady flows, is applied to investigate the stability of an evaporating droplet against disturbances of any amplitude.

The results predicted by the present study possess similar trends with those acquired by the linear stability analysis. Both studies indicate that, as time proceeds, both the increase of the surface temperature reduction and the growth of the thermal boundary layer near the free surface are conducive to the onset of instability. The critical Marangoni number and wave number increase with the droplet initial temperature. For all the cases studied, the critical Marangoni numbers predicted by the energy method are smaller than those calculated by the linear stability analysis. However, the subcritical region between the $M a_{\mathrm{c}}$ 's as calculated by these two methods is very narrow. This consistency indicates the validity of linear stability analysis as a first approach to the analysis of Marangoni instability of an evaporating droplet.

(c) 2004 Elsevier Ltd. All rights reserved.
\end{abstract}

Keywords: Marangoni instability; Evaporating droplet; Energy method

\section{Introduction}

Conventional hydrodynamic stability theory is mainly concerned with the determination of the critical values of parameters, such as the critical Reynolds number, Rayleigh number or Taylor number, demarcating a region of stability from that of instability. The theory based on small disturbances superimposed on a basic flow is called the linearized stability theory. Because of its simplicity and straightforwardness, the linearized stability theory is generally applied to analyze the instability of a given flow. However, suffering from

\footnotetext{
${ }^{*}$ Corresponding author. Tel.: +886-2-2363-4820; fax: +8862-2363-1755.

E-mail address: cllai@ntu.edu.tw (C.-L. Lai).
}

its basic assumption, the validity of the linearized stability theory becomes questionable when applied to an unsteady flow or disturbances of finite strength. Hence, the non-linear approach becomes inevitable to investigate the effects of finite disturbances.

The oldest method of non-linear stability analysis which can deal with finite disturbances is the energy method, originated by Reynolds [2] and Orr [3]. They found the critical Reynolds numbers for simple perturbed flows with an equation related to the global kinetic energy. However, these early results provided quite conservative estimates for the critical Reynolds number. The energy method was thus discarded for years. Later, Serrin [4] and Joseph [5,6] reformulated the energy method, and two important results were deduced from the investigation of the stability of viscous fluid motions. The first was the existence of a universal stability criterion for 


\begin{tabular}{|c|c|c|c|}
\hline \multicolumn{4}{|c|}{ Nomenclature } \\
\hline$B i$ & heat exchange parameter & $U$ & magnitude of the perturbed $R$-component \\
\hline $\mathrm{Cr}$ & Crispation number & & velocity \\
\hline$D$ & mass diffusivity & $u$ & velocity component \\
\hline $\mathscr{D}$ & integrals with the definition as Eq. (3.5b) & $\vec{V}$ & velocity vector \\
\hline$E$ & $\begin{array}{l}\text { a positive-definite energy function with the } \\
\text { definition } E=K+\lambda M a \Theta\end{array}$ & $\begin{array}{l}\mathscr{V} \\
Y_{\mathrm{v}}\end{array}$ & $\begin{array}{l}\text { space occupied by the droplet } \\
\text { mass fraction of the liquid vapor }\end{array}$ \\
\hline$E v$ & dimensionless evaporation rate & $Y_{1}^{m}$ & spherical harmonics \\
\hline $\mathscr{H}$ & $\begin{array}{l}\text { set of suitably differential functions } \vec{V}^{\prime} \text { and } \Phi \\
\text { with the definition as Eq. (3.6b) }\end{array}$ & \multicolumn{2}{|c|}{ Greek symbols } \\
\hline$h_{\mathrm{lg}}$ & latent heat of evaporation & $\alpha$ & thermal diffusivity \\
\hline $\mathscr{I}_{\lambda}$ & integrals with the definition as Eq. $(3.5 \mathrm{c})$ & $\delta$ & notation of the variation operator \\
\hline$i$ & imaginary unit & $\Theta$ & quantity scaling the magnitude of changes in \\
\hline$K$ & $\begin{array}{l}\text { the total kinetic energy of the perturbed field } \\
\text { in the evaporating droplet }\end{array}$ & $\mu$ & $\begin{array}{l}\text { the total thermal energy of the perturbed } \\
\text { field in the evaporating droplet } \\
\text { viscosity }\end{array}$ \\
\hline$k$ & thermal conductivity & $v^{\mu}$ & kinematic viscosity \\
\hline Le & Lewis number & 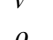 & density \\
\hline$L^{2}$ & operator & $\sigma$ & surface tension \\
\hline$l$ & wave number of the disturbances & $\tau$ & dimensionless time \\
\hline$\frac{M a}{\widetilde{M} a_{\mathrm{c}}}$ & $\begin{array}{l}\text { Marangoni number } \\
\text { maximum value of the specified definite } \\
\text { integrals with its definition as Eq. (3.6a) }\end{array}$ & $\Phi$ & $\begin{array}{l}\text { redefined dimensionless temperature with } \\
\text { the definition } \Phi=\sqrt{\lambda M a} T^{\prime}\end{array}$ \\
\hline$M a_{\lambda}$ & $\begin{array}{l}\text { eigenvalues of the Euler-Lagrange equa- } \\
\text { tions }(2.38),(2.41),(2.42),(3.9)-(3.16)\end{array}$ & $\begin{array}{l}\phi_{k} \\
\phi_{\alpha}\end{array}$ & $\begin{array}{l}\text { thermal conductivity ratio } \\
\text { thermal diffusivity ratio }\end{array}$ \\
\hline$m$ & azimuthal wave number & & $\begin{array}{l}\text { viscosity ratio } \\
\text { density ratio }\end{array}$ \\
\hline$m^{\prime}$ & dimensional evaporation rate & & defined parameter \\
\hline $\begin{array}{l}P_{1}^{m} \\
P r\end{array}$ & associated Legendre polynomials & & transfer due to temperature perturbation \\
\hline $\begin{array}{l}P r \\
p\end{array}$ & $\begin{array}{l}\text { Prandtl number } \\
\text { pressure }\end{array}$ & \multicolumn{2}{|c|}{ Miscellaneous symbols } \\
\hline$Q$ & $\begin{array}{l}\text { dimensionless heat flux due to the evapora- } \\
\text { tion }\end{array}$ & $\stackrel{\mathfrak{R}}{\mathfrak{J}}$ & $\begin{array}{l}\text { universal gas constant } \\
\text { magnitude of temperature perturbation }\end{array}$ \\
\hline $\begin{array}{l}R, \theta, \phi \\
\dot{R}\end{array}$ & $\begin{array}{l}\text { dimensionless spherical coordinates } \\
\text { regression rate of the evaporating free sur- } \\
\text { face }\end{array}$ & \multicolumn{2}{|c|}{ Superscripts } \\
\hline$r, \theta, \phi$ & dimensional spherical coordinates & $*$ & dimensionless quantity \\
\hline$r_{0}$ & radius of the evaporating droplet & \multicolumn{2}{|c|}{ Subscripts } \\
\hline$r_{\mathrm{w}}$ & ratio of molecular weights $M_{\mathrm{amb}} / M_{1}$ & $\mathrm{c}$ & critical condition \\
\hline $\mathscr{S}$ & the evaporating free surface of the droplet & $\mathrm{g}$ & gas property \\
\hline$T$ & temperature & 1 & liquid property \\
\hline $\mathscr{T}$ & $\begin{array}{l}\text { set of suitably differential functions } \vec{V}^{\prime} \text { and } \Phi \\
\text { with the similar definition of } \mathscr{H} \text { except the } \\
\text { continuity condition being removed } \\
\text { dimensional time }\end{array}$ & $\begin{array}{l}\mathrm{v} \\
\mathrm{s} \\
0\end{array}$ & $\begin{array}{l}\text { vapor property (of the evaporating liquid) } \\
\text { surface condition } \\
\text { initial condition }\end{array}$ \\
\hline
\end{tabular}

flows with arbitrary disturbances in a bounded region of space. The other was the possibility of an improved stability criterion obtained by variational method. With this reformulation, the energy method became successful in providing sufficient conditions for stability of a large number of steady and unsteady flows.

In contrast to the time-independent flow, the analysis of the instability of a time varying flow is much more complicated. Not only the critical value of the relevant parameter, but also the onset time and the growth rate of the disturbances are very important. Early approaches to the problem consisted of the quasi-steady analysis (freezing the basic state at a given instant and determining the marginal instability) and the initialvalue method (specifying an initial distribution of disturbances in the flow field and computing their evolution 
with time by integrating the perturbed equations). However, the validity of the quasi-steady assumption on time-varying flows is questionable. The initial-value method depends strongly on the initial data specified and the results calculated disagree, in general, with the experimental observations. Moreover, these approaches are valid only for infinitesimal disturbances. To resolve these deficiencies, the energy method seems to be very promising.

Homsy [7] employed the energy method (corrected later by Neitzel [8]) to analyze the stability of a liquid layer heated or cooled impulsively. The region of finiteamplitude convection was demarcated in a Rayleigh number-time plane. Later, Davis and von Kerczek [9] presented a reformulation of the energy method and the improved algorithm could yield an exponential decay of disturbances without the usage of the isoperimetric inequality (Joseph [5,6]). Hence, the stability of a boundary-layer type flow, of which the domain was not finite in at least one direction (e.g. jets, wakes), could then be examined. There were two stability criteria defined previously. One was referred to as the strong global stability if the energy of the disturbances decayed exponentially in the mean. The other was referred to as the marginal stability if the energy of the disturbances was less than or equal to its initial value during the time of interest. Homsy [10] and Gumerman et al. [11] followed the new approach of energy method to seek the strong stability and marginal stability criteria for Benard problems subject to modulations in either the surface temperature or gravity, and a flow driven by surface tension, respectively. David and Homsy [12] then generalized the energy method to analyze the stability of a buoyancy-thermocapillary layer with a deformable free surface. The Euler-Lagrange equations obtained were strongly non-linear. Only certain limiting cases with small capillary number were studied. The authors suggested that the method could be further generalized to deal with problems involving three-dimensional disturbances, non-Newtonion fluids and consideration of surface viscosity and elasticity. Furthermore, Neitzel and Davis [13] and Neitzel [14] determined the sufficient conditions for stability and predicted the onset time of instability of a decelerating swirl flow. Neitzel [15] analyzed the instability of a circular Couette flow with a variable inner cylinder speed. Consistency between the results obtained, respectively, by the energy method and the linear stability analysis were found.

As a powerful tool for the analysis of unsteady flows, the energy method is employed in the present study to seek the stability boundary of an evaporating droplet. The mechanism for the onset of instability induced by the surface tension in an evaporating liquid is similar to that of the classical Marangoni instability. A condition of constant heat flux from the free surface is usually used to model the evaporation. Many studies have been made to analyze the instability of a planar liquid layer suddenly cooled from above or heated from below [1,7,8,16-30]. Much less work has been done on the onset of Marangoni instability in a spherical geometry.

With a constant heat and mass flux, the instability of a deformable spherical droplet suspended in another immiscible liquid was investigated by Sorensen et al. [31] using linear stability analysis. They claimed that the results might serve as a starting point for the understanding of the interfacial turbulence and the deformation of cell membrane. Higuera and Linan [32] considered the linear stability problem of a droplet vaporizing in hot atmosphere. The Marangoni effect was stabilizing and the viscous stresses effect was destabilizing. Pirotte and Lebon [33] analyzed the Marangoni instability of a spherical liquid layer with an undeformable free surface. The critical Marangoni number obtained was higher than that of the planar case [1]. They claimed that it was mainly due to the existence of surface curvature. Investigations were also focused on the problem of instability in a spherical liquid layer with a non-deformable or deformable free surface [34-38]. Chai et al. [39] demonstrated that, with an approximate dimensional analysis and some experimental results, Marangoni instability induced convection could occur in an evaporating liquid droplet. A review of recent work concerning the Marangoni instability in a spherical geometry can be found in the research by Lebon et al. [40]. Wilson [41] extended the earlier work of Pirotte and Lebon [33] to include the effect of a deformable free surface. Errors in the Cloot and Lebon's work [35] in formulating the boundary conditions were corrected. Their results indicated that, if the free surface of the layer was non-deformable, the layer was always stable when heated from the outside. It was unstable when heated from the inside if the (positive) Marangoni number was sufficiently large. However, if the free surface of the layer was deformable, the neutral curves were characterized by the bifurcation at $\mathrm{Cr}=r_{2} / 4$, where $\mathrm{Cr}$ is the Crispation number and $r_{2}$ is the dimensionless radius of the undisturbed outer surface of the spherical shell of liquid. If $\mathrm{Cr}>r_{2} / 4$, the layer was possibly unstable when heated from either inside or outside. Ha [19] and Ha and Lai [20] modelled a configuration similar to that of Wilson. The effect of phase change during the evaporation process was considered by simultaneously solving the energy and species equations. With the quasi-steady assumption, the sufficient conditions for the onset of Marangoni instability of an evaporating droplet were determined. The results indicated that, for a non-deformable droplet, both the critical Marangoni number and the critical wave number increased with the initial temperature and decreased with time. No oscillatory instability was found within the parametric ranges of interest. For a droplet with a deformable free surface, 
the stationary instability is still the preferred mode of instability. Generally, surface deformation is a destabilizing factor.

In the above studies, the linear stability analysis was applied to investigate the onset of Marangoni instability in a spherical geometry. As a result, they are only valid for steady (quasi-steady) flows with small disturbances.

The present study is therefore aimed at resolving these deficiencies by the energy method and investigating the stability of an evaporating droplet subject to disturbances of any amplitude. The onset and physical mechanism of Marangoni instability of an evaporating droplet are thence justified and demonstrated clearly. The basic flow is solved first. The global energy equation of the disturbances is derived and the stability criteria are then derived. Comparison of the results obtained, respectively, by the energy method and the linear stability analysis will be discussed finally.

\section{Mathematical formulation}

\subsection{The governing equations and boundary conditions}

The system to be considered is a motionless droplet of radius $r_{0}$ surrounded by a passive gas with an initial mass fraction $Y_{\infty}$ of the evaporating component and at temperature $T_{0}$ and pressure $p_{\infty}$. The droplet is originally at pressure $p_{0}$ and the same temperature $T_{0}$ as the surrounding gas. A schematic diagram of the physical model is shown in Fig. 1.

As shown by Ha and Lai [20] through scaling analysis, when the interest is mainly on the liquid motion of the droplet, the surrounding gas motion can be treated as asymptotic steady and the free surface regression can

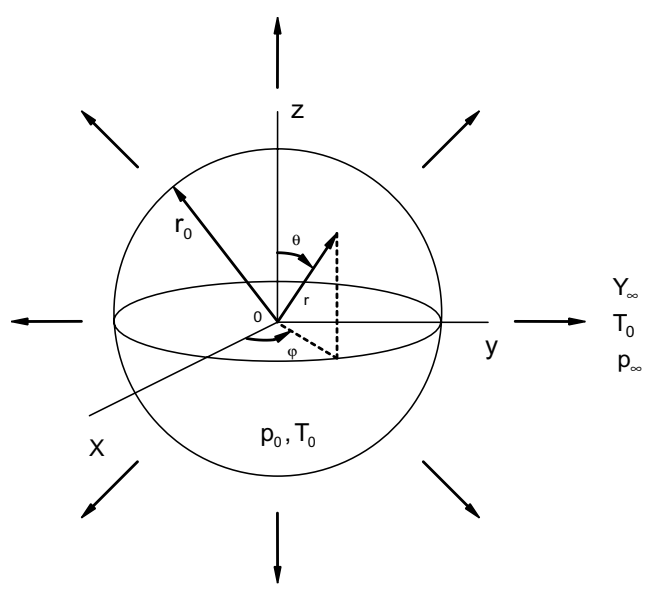

Fig. 1. The schematic diagram of the physical model and coordinate system for an evaporating liquid droplet. be neglected. Further assumptions are incompressible flow with constant physical properties except the surface tension which is a monotonically decreasing function of temperature, non-deformable free surface, and negligible gravity effects. By introducing the scaling factors for length $\left(r_{0}\right)$, time $\left(r_{0}^{2} / \alpha_{1}\right)$, velocity $\left(\alpha_{1} / r_{0}\right)$, temperature $\left(m_{0}^{\prime} h_{\mathrm{lg}} r_{0} / k_{1}\right)$ with $m_{0}^{\prime}$ denoting the initial evaporation rate at the droplet surface and $h_{\mathrm{lg}}$, the latent heat of evaporation at $T_{0}$, pressure $\left(\rho_{1} \alpha_{1}^{2} / r_{0}^{2}\right)$, and mass fraction $\left(Y_{0}\right)$, the dimensionless governing equations become, as derived by $\mathrm{Ha}$ and Lai [20],

$$
\begin{aligned}
& \nabla \cdot \vec{V}_{\mathrm{g}}^{*}=0, \\
& \nabla \cdot \vec{V}_{1}^{*}=0, \\
& \frac{\phi_{\alpha}}{P r_{\mathrm{g}}}\left(\vec{V}_{\mathrm{g}}^{*} \cdot \nabla \vec{V}_{\mathrm{g}}^{*}+\phi_{\rho} \nabla p_{\mathrm{g}}^{*}\right)=\nabla^{2} \vec{V}_{\mathrm{g}}^{*}, \\
& \frac{1}{\operatorname{Pr}_{\mathrm{l}}}\left(\frac{\partial \vec{V}_{1}^{*}}{\partial \tau}+\vec{V}_{1}^{*} \cdot \nabla \vec{V}_{1}^{*}+\nabla p_{1}^{*}\right)=\nabla^{2} \vec{V}_{1}^{*}, \\
& \phi_{\alpha}\left(\vec{V}_{\mathrm{g}}^{*} \cdot \nabla T_{\mathrm{g}}^{*}\right)=\nabla^{2} T_{\mathrm{g}}^{*}, \\
& \frac{\partial T_{1}^{*}}{\partial \tau}+\vec{V}_{1}^{*} \cdot \nabla T_{1}^{*}=\nabla^{2} T_{1}^{*}, \\
& \phi_{\alpha} L e\left(\vec{V}_{\mathrm{g}}^{*} \cdot \nabla Y_{\mathrm{v}}^{*}\right)=\nabla^{2} Y_{\mathrm{v}}^{*} .
\end{aligned}
$$

In the above equations, $\tau$ is time, $\vec{V}$ is the velocity vector, $T$ is the temperature, $p$ denotes the pressure, and $Y_{\mathrm{v}}$ represents the mass fraction of the liquid vapor and is described by the Clausius-Clapeyron relation. $\phi_{\alpha}=\frac{\alpha_{1}}{\alpha_{\mathrm{g}}}$ is the thermal diffusivity ratio and $\phi_{\rho}=\frac{\rho_{1}}{\rho_{\mathrm{g}}}$ is the density ratio. $\operatorname{Pr}=\frac{v}{\alpha}$ is Prandtl number and $L e=\frac{\alpha}{D}$ is Lewis number. Subscripts $g$ and 1 refer to the gas phase and liquid phase, respectively, and superscript * indicates that the physical quantity is dimensionless.

The initial conditions at $\tau=0$ are

$\vec{V}_{1}^{*}=0, \quad T_{1}^{*}=0$.

As $R \rightarrow \infty$, the solutions must satisfy

$\vec{V}_{\mathrm{g}}^{*}=0, \quad T_{\mathrm{g}}^{*}=0$,

$p_{\mathrm{g}}^{*}=p_{\infty}^{*}, \quad Y_{\mathrm{v}}^{*}=Y_{\infty}^{*}=0$.

At the center of the droplet, $R=0$, the boundary conditions are

$\vec{V}_{1}^{*}=0, \quad T_{1}^{*}=$ finite.

At the droplet surface, $R=1$, the continuity of velocity, mass flux, temperature and heat flux, the non-condensible condition of the passive gas and the balance of forces must be satisfied. They are expressed as follows.

$u_{\theta, 1}^{*}=u_{\theta, \mathrm{g}}^{*}, \quad u_{\phi, 1}^{*}=u_{\phi, \mathrm{g}}^{*}$, 


$$
\begin{aligned}
& u_{R, 1}^{*}-\dot{R}=\frac{1}{\phi_{\rho}}\left(u_{R, \mathrm{~g}}^{*}-\dot{R}\right)=E v, \\
& T_{\mathrm{g}}^{*}=T_{1}^{*}=T_{\mathrm{s}}^{*}, \\
& -\frac{\partial T_{1}^{*}}{\partial R}=-\frac{1}{\phi_{k}} \frac{\partial T_{\mathrm{g}}^{*}}{\partial R}+\frac{m^{\prime}}{m_{0}^{\prime}}, \\
& \frac{1}{\phi_{\alpha} L e} \frac{\partial Y_{\mathrm{v}}^{*}}{\partial R}=-\left(\frac{1}{Y_{0}}-Y_{\mathrm{v}}^{*}\right) u_{R, \mathrm{~g}}^{*}, \\
& -\phi_{\mu}\left[R \frac{\partial}{\partial R}\left(\frac{u_{\theta, 1}^{*}}{R}\right)+\frac{1}{R} \frac{\partial u_{R, 1}^{*}}{\partial \theta}\right]+\left[R \frac{\partial}{\partial R}\left(\frac{u_{\theta, \mathrm{g}}^{*}}{R}\right)+\frac{1}{R} \frac{\partial u_{R, \mathrm{~g}}^{*}}{\partial \theta}\right] \\
& \quad=\frac{\phi_{\mu} M a}{R} \frac{\partial T^{*}}{\partial \theta}, \\
& -\phi_{\mu}\left[\frac{1}{R \sin \theta} \frac{\partial u_{R, 1}^{*}}{\partial \phi}+R \frac{\partial}{\partial R}\left(\frac{u_{\phi, 1}^{*}}{R}\right)\right] \\
& \quad+\left[\frac{1}{R \sin \theta} \frac{\partial u_{R, \mathrm{~g}}^{*}}{\partial \phi}+R \frac{\partial}{\partial R}\left(\frac{u_{\phi, \mathrm{g}}^{*}}{R}\right)\right] \\
& \quad=\frac{\phi_{\mu} M a}{R \sin \theta} \frac{\partial T^{*}}{\partial \phi}, \\
& \left(-p_{1}^{*}+2 P r_{1} \frac{\partial u_{R, 1}^{*}}{\partial R}\right)-\left(-p_{\mathrm{g}}^{*}+\frac{2 P_{r_{\mathrm{g}}}}{\phi_{\alpha} \phi_{\rho}} \frac{\partial u_{R, \mathrm{~g}}^{*}}{\partial R}\right) \\
& \quad=-2 \frac{P r_{1}}{C r},
\end{aligned}
$$

where $\phi_{k}=\frac{k_{1}}{k_{\mathrm{g}}}$ is the heat conductivity ratio and $\phi_{\mu}=\frac{\mu_{1}}{\mu_{\mathrm{g}}}$ is the viscosity ratio. $E v=\frac{m^{\prime} r_{0}}{\rho_{1} \alpha_{1}}$ is the dimensionless evaporation rate per unit area per unit time at the free surface. The expression for $E v$ will be obtained later by using the velocity field of the gas phase. $C r=\frac{\mu_{1} \alpha_{1}}{\sigma_{0} r_{0}}$ is the Crispation number with $\sigma_{0}$ being the surface tension at $T_{0}$. $M a=-\frac{(\partial \sigma / \partial T) m_{0}^{\prime} h_{\lg } r_{0}^{2}}{\mu_{1} \alpha_{1} k_{1}}$ is the Marangoni number. $\dot{R}$ is the regression rate of the droplet which will be neglected in the later analysis while is still retained here for the sake of mass balance.

\subsection{The basic flow}

Since the attention is focused on the evaporation of a quiescent droplet, no motion occurs in the liquid phase. The pressure in the droplet is then constant and can be determined from the normal-force balance condition at the surface, Eq. (2.24), as follows

$$
\begin{aligned}
p_{1}^{*}= & \frac{2 P r_{1}}{C r}-\frac{4 P r_{\mathrm{g}}}{\phi_{\alpha}^{2} \phi_{\rho} L e} \ln \left(1-Y_{\mathrm{v}}(1)\right) \\
& -\frac{1}{2 \phi_{\rho} \phi_{\alpha}^{2} L e^{2}} \ln ^{2}\left(1-Y_{\mathrm{v}}(1)\right)+p_{\infty}^{*} .
\end{aligned}
$$

However, the evaporation will result in an outward flow motion away from the droplet surface in the gas phase. The radial velocity, pressure, temperature, and liquid vapor distribution in the surrounding gas phase can be derived analytically in the following forms from the corresponding equations and boundary conditions [20].

$u_{R, \mathrm{~g}}^{*}=-\frac{\ln \left(1-Y_{\mathrm{v}}(1)\right)}{\phi_{\alpha} L e} \frac{1}{R^{2}}$,

$p_{\mathrm{g}}^{*}=-\frac{\ln ^{2}\left(1-Y_{\mathrm{v}}(1)\right)}{\phi_{\alpha}^{2} \phi_{\rho} L e^{2}} \frac{1}{2 R^{4}}+p_{\infty}^{*}$,

$T_{\mathrm{g}}^{*}=T_{\mathrm{s}}^{*} \frac{\left(1-Y_{\mathrm{v}}(1)\right)^{\frac{1}{L e \cdot R}}-1}{\left(1-Y_{\mathrm{v}}(1)\right)^{\frac{1}{L e}}-1}$,

$Y_{\mathrm{v}}^{*}=\frac{1}{Y_{0}}\left[1-\left(1-Y_{\mathrm{v}}(1)\right)^{\frac{1}{R}}\right]$,

where

$$
Y_{\mathrm{v}}(1)=\frac{\exp \left[\frac{h_{\mathrm{fg}}}{\mathfrak{R}}\left(\frac{1}{T_{\mathrm{b}}}-\frac{1}{T_{\mathrm{s}}}\right)\right]}{r_{\mathrm{w}}-\left(r_{\mathrm{w}}-1\right) \exp \left[\frac{h_{\mathrm{fg}}}{\mathfrak{R}}\left(\frac{1}{T_{\mathrm{b}}}-\frac{1}{T_{\mathrm{s}}}\right)\right]}
$$

is the mass fraction of the liquid vapor at the droplet surface. The dimensionless evaporation rate is then determined by the continuity condition of mass flux, Eq. (2.18), i.e.,

$E v=-\frac{1}{L e \phi_{\alpha}\left(\phi_{\rho}-1\right)} \ln \left(1-Y_{\mathrm{v}}(1)\right)$.

The temperature field of the droplet, $T_{1}^{*}(R, \tau)$, cannot be solved easily in an analytic form and has to be numerically calculated from the following energy equation and conditions [20]:

$\frac{\partial T_{1}^{*}}{\partial \tau}=\frac{1}{R^{2}} \frac{\partial}{\partial R}\left(R^{2} \frac{\partial T_{1}^{*}}{\partial R}\right)$,

$\tau=0, \quad T_{1}^{*}=0$,

$R=0, \quad T_{1}^{*}=$ finite,

$R=1, \quad-\frac{\partial T_{1}^{*}}{\partial R}=T_{\mathrm{s}}^{*} B i+Q$.

In Eq. (2.34), the first term of the right hand side represents the heat transfer to the ambient by convection and the second term denotes the heat flux due to evaporation at the free surface, with the parameters defined as

$$
\begin{aligned}
& B i=\frac{1}{\phi_{k} L e} \ln \left(1-Y_{\mathrm{v}}(1)\right) \frac{\left(1-Y_{\mathrm{v}}(1)\right)^{\frac{1}{L e}}}{\left(1-Y_{\mathrm{v}}(1)\right)^{\frac{1}{L e}}-1}, \\
& Q=\frac{m^{\prime}}{m_{0}^{\prime}}=\frac{\ln \left(1-Y_{\mathrm{v}}(1)\right)}{\ln \left(1-Y_{0}(1)\right)} .
\end{aligned}
$$

The transient temperature distributions of the droplet are solved numerically by using the Crank-Nicolson method, which is of second-order accuracy $\left[(\Delta \tau)^{2}\right.$, $\left.(\Delta R)^{2}\right]$. To approximate the true transient solution, the quantities $\Delta \tau$ and $\Delta R$ have been chosen in such a way 
that the discrete solution must converge to within a certain accuracy, say $10^{-4}$. Moreover, a non-uniform grid distribution in the radial direction is used and the grid points are clustered in the vicinity of free surface; hence, both the accuracy and efficiency of computation are enhanced. The algebraic expression relating the grid distribution in the computational domain to that in the physical domain is referred to the book written by Hoffmann [42]. The computations are then performed by using a non-uniform grid of 1000 points and a time step of $\Delta \tau=10^{-7}$, and are accurate to the fourteenth decimal with the double-precision arithmetic on PC. Within the thermal boundary layer, at least 130 grid points are used to calculate the temperature variation with time. With the equation of $Y_{\mathrm{v}}(1)$, all physical variables of the gas phase, which are expressed in terms of $T_{\mathrm{s}}^{*}$, can then be determined.

All the physical variables of the gas phase which are expressed in terms of the surface temperature $T_{\mathrm{s}}^{*}$ can then be determined.

\subsection{The disturbance equations}

The stability of the basic flow is investigated by superposing disturbances on the velocity, temperature and pressure fields. Let

$\vec{V}=0+\vec{V}^{\prime}(R, \theta, \phi, \tau)$

$T=T^{*}(R, \tau)+T^{\prime}(R, \theta, \phi, \tau)$,

$p=p^{*}(R, \tau)+p^{\prime}(R, \theta, \phi, \tau)$,

the perturbed flow fields must then satisfy the following equations

$\nabla \cdot \vec{V}^{\prime}=0$

$\frac{1}{\operatorname{Pr}}\left(\frac{\partial \vec{V}^{\prime}}{\partial \tau}+\vec{V}^{\prime} \cdot \nabla \vec{V}^{\prime}\right)=-\frac{1}{\operatorname{Pr}} \nabla p^{\prime}+\nabla^{2} \vec{V}^{\prime}$,

$\frac{\partial T^{\prime}}{\partial \tau}+\vec{V}^{\prime} \cdot \nabla T^{\prime}=\nabla^{2} T^{\prime}-\vec{V}^{\prime} \cdot \nabla T^{*}$,

where the superscript "'" ' is used to denote the disturbances while $l$, used for the liquid phase previously, is omitted for simplification.

The boundary conditions at the center of the droplet are

$\vec{V}^{\prime}=0, \quad T^{\prime}=$ finite

On the free surface, the boundary conditions are

$u_{R}^{\prime}=\frac{\partial E v}{\partial T^{*}} T^{\prime}$,

$R \frac{\partial}{\partial R}\left(\frac{u_{\theta}^{\prime}}{R}\right)+\frac{1}{R} \frac{\partial u_{R}^{\prime}}{\partial \theta}=-\frac{M a}{R} \frac{\partial T^{\prime}}{\partial \theta}$,

$$
\begin{aligned}
& \frac{1}{R \sin \theta} \frac{\partial u_{R}^{\prime}}{\partial \phi}+R \frac{\partial}{\partial R}\left(\frac{u_{\phi}^{\prime}}{R}\right)=-\frac{M a}{R \sin \theta} \frac{\partial T^{\prime}}{\partial \phi}, \\
& -p^{\prime}+2 \operatorname{Pr} \frac{\partial u_{R}^{\prime}}{\partial R}=\frac{2}{R}{\operatorname{Pr} M a T^{\prime}}^{\prime} \\
& -\frac{\partial T^{\prime}}{\partial R}=\left(B i+\frac{\partial B i}{\partial T^{*}} T^{*}+\frac{\partial Q}{\partial T^{*}}\right) T^{\prime}=\chi T^{\prime} .
\end{aligned}
$$

Eqs. (2.43)-(2.47) are, respectively, the perturbed boundary conditions satisfying the continuity of mass flux, shear stresses in $\theta$ - and $\phi$-directions, normal stress, and heat flux. Eqs. (2.38)-(2.47) are to be analyzed by the energy method to determine the criteria of Marangoni instability of an evaporating droplet.

\section{Energy stability analysis}

\subsection{The energy identities}

At instant $\tau=0$, there is no flow in the droplet and the temperature reduction near the free surface starts to develop due to evaporation. As time proceeds, the temperature reduction continues to develop and, in the mean time, disturbances might be introduced. The evolution of the disturbances is the main concern of stability analysis, and in the present study the energy method is used for this purpose.

Before the formal formulation of the energy method, the kinetic energy of the perturbed flow motion, $K$, and a quantity $\Theta$ are introduced first:

$K=\int_{\mathscr{r}}\left(\frac{\left|\vec{V}^{\prime}\right|^{2}}{2}\right)$ and $\Theta=\int_{\mathscr{r}}\left(\frac{T^{\prime^{2}}}{2}\right)$.

The quantity $\Theta$ scales the magnitude of thermal energy of the perturbed temperature field and $\mathscr{V}$ refers to the space occupied by the droplet. If both $K$ and $\Theta$ tend to vanish as $\tau \rightarrow \infty$, the basic flow is stable; otherwise, instability might occur eventually.

By taking the dot product of Eq. (2.39) with $\vec{V}^{\prime}$ and the scalar product of Eq. (2.40) with $T^{\prime}$, and then integrating, respectively, over the entire region $\mathscr{V}$ with the aid of the continuity equation (2.38), the equations determining the rates of change of $K$ and $\Theta$ are derived:

$$
\begin{aligned}
\frac{1}{\operatorname{Pr}} \frac{\mathrm{d} K}{\mathrm{~d} \tau}= & \frac{1}{\operatorname{Pr}} \frac{\mathrm{d}}{\mathrm{d} \tau}\left\langle\frac{\left|\vec{V}^{\prime}\right|^{2}}{2}\right\rangle \\
= & -\frac{1}{\operatorname{Pr}}\left\langle\nabla \cdot\left(\vec{V}^{\prime} p^{\prime}\right)\right\rangle+\left\langle\nabla \cdot\left(\vec{V}^{\prime} \times \nabla \times \vec{V}^{\prime}\right)\right\rangle \\
& -\left\langle\left(\nabla \times \vec{V}^{\prime}\right) \cdot\left(\nabla \times \vec{V}^{\prime}\right)\right\rangle, \\
\frac{\mathrm{d} \Theta}{\mathrm{d} \tau}= & \left\langle\nabla \cdot \frac{\nabla T^{\prime 2}}{2}\right\rangle-\left\langle\nabla T^{\prime} \cdot \nabla T^{\prime}\right\rangle-\left\langle\vec{V}^{\prime} T^{\prime} \cdot \nabla T^{*}\right\rangle .
\end{aligned}
$$


In the above equations, \langle\rangle denotes volume integration over the region $\mathscr{V}$. By taking a linear combination of Eqs. (3.3) and (3.4) to form a positive-definite energy functional $E=K+\lambda M a \Theta$ with $\lambda, M a>0$, and employing the divergence theorem and relevant boundary conditions, the following energy evolution equation is derived:

$\frac{\mathrm{d} E}{\mathrm{~d} \tau}=-\mathscr{D}+M a^{1 / 2} \mathscr{I}_{\lambda}$

where

$$
\begin{aligned}
\mathscr{D}= & \left\langle\left(\nabla \times \vec{V}^{\prime}\right) \cdot\left(\nabla \times \vec{V}^{\prime}\right)\right\rangle+\langle\nabla \Phi \cdot \nabla \Phi\rangle \\
& +\int_{\mathscr{S}} \chi \Phi^{2} \mathrm{~d} S+2 \int_{\mathscr{S}} u_{R}^{\prime} \frac{\partial u_{R}^{\prime}}{\partial R} \mathrm{~d} S \\
& -2 \int_{\mathscr{S}}\left(\frac{u_{\theta}^{\prime 2}}{R}-\frac{u_{\theta}^{\prime}}{R} \frac{\partial u_{R}^{\prime}}{\partial \theta}+\frac{u_{\phi}^{\prime 2}}{R}-\frac{u_{\phi}^{\prime}}{R \sin \theta} \frac{\partial u_{R}^{\prime}}{\partial \phi}\right) \mathrm{d} S,
\end{aligned}
$$

$$
\begin{aligned}
\mathscr{I}_{\lambda}= & -\lambda^{1 / 2}\left\langle\vec{V}^{\prime} \Phi \cdot \nabla T^{*}\right\rangle-\lambda^{-1 / 2} \int_{\mathscr{S}}\left(\frac{u_{\theta}^{\prime}}{R} \frac{\partial \Phi}{\partial \theta}+\frac{u_{\phi}^{\prime}}{R \sin \theta} \frac{\partial \Phi}{\partial \phi}\right) \mathrm{d} S \\
& +2 \lambda^{-1 / 2} \int_{\mathscr{S}} u_{R}^{\prime} \frac{\Phi}{R} \mathrm{~d} S
\end{aligned}
$$

with $\Phi=\sqrt{\lambda M a} T^{\prime}$ as the dependent variable. Symbol $\mathscr{S}$ denotes the evaporating surface of the droplet. Knowing the basic state of the temperature field, the stability criteria can then be determined from Eqs. (3.5a)-(3.5c).

\subsection{The strong global stability}

The flow is said to be strongly stable $[6,10]$ if $M a<\widetilde{M a}$, where

$\widetilde{M a_{\mathrm{c}}^{-1 / 2}}=\max _{\mathscr{H}} \frac{\mathscr{I}_{\lambda}}{\mathscr{D}} \quad$ with $\mathscr{D}=1$,

and

$\mathscr{H}=\left\{\left(\vec{V}^{\prime}, \Phi\right) \mid \nabla \cdot \vec{V}^{\prime}=0, \vec{V}^{\prime}=0\right.$,

$\Phi=$ finite at $R=0$,

$M a^{1 / 2} \lambda^{1 / 2} u_{R}^{\prime}=\frac{\partial E v}{\partial T^{*}} \Phi \quad$ at $\left.R=1\right\}$.

$\mathscr{H}$ represents the set of suitably differentiable functions $\vec{V}^{\prime}$ and $\Phi$. It can be shown that, for $M a<\tilde{M} a_{\mathrm{c}}$, the integrated energy function $E$ satisfies the inequality $E^{-1}\left(\frac{\mathrm{d} E}{\mathrm{~d} \tau}\right) \leqslant 0$, implying an energy decay. This inequality is valid for any given $\lambda>0$. Hence, it leaves $\lambda$ as a free parameter which can then be chosen to best extend the limit for stability, i.e., to obtain the greatest value of Marangoni number $M a_{\mathrm{c}}$ below which the energy decreases exponentially. Analytically, the best value of $\lambda$ is found such that
$M a_{\mathrm{c}}=\max _{\lambda>0} \widetilde{M} a_{\mathrm{c}}$

and $M a<M a_{\mathrm{c}}$ is a sufficient condition for stability regardless of the magnitude of disturbances.

Since $\lambda$ is chosen to yield the largest eigenvalue, it may actually vary with time. Strictly speaking, Eq. (3.5a) is derived under the assumption that $\lambda$ is a constant. However, Homsy [7] has showed that, for $\mathrm{d} \lambda / \mathrm{d} t \leqslant 0$, i.e. $\lambda$ being a monotonically decreasing function of time, the derivation still hold. He also claimed that, for the cases when $M a_{\mathrm{c}}$ varied monotonically with time, the optimal stability boundary remained the same even if $\mathrm{d} \lambda / \mathrm{d} t>0$. Neitzel [8] reported that there existed cases where $M a_{\mathrm{c}}$ did not decrease with time monotonically (i.e. the stability curve reached a minimum first and then began to increase toward a steady-state limit) and care had to be taken for this situation. He suggested that it was much better to choose $\lambda$ on the decreasing portion of the curve. For an evaporating droplet, it will be shown later that the stability curve decreases with time only.

Then, Lagrange multipliers $M a_{\lambda}$ and $\Pi(R, \theta, \phi)$ are introduced and the system (3.6) can be reformulated as a system of partial differential equations by requiring

$\delta\left\{\mathscr{I}_{\lambda}+2 \frac{\Pi}{{\operatorname{Pr} M a_{\lambda}^{1 / 2}}_{2}}\left\langle\nabla \cdot \vec{V}^{\prime}\right\rangle-\frac{1}{M a_{\lambda}^{1 / 2}} \mathscr{D}\right\}=0$.

The Euler-Lagrange equations corresponding to the variational problem, Eq. (3.8), are

$\nabla \cdot \vec{V}^{\prime}=0$

$-\frac{1}{\operatorname{Pr}} \nabla \Pi+\nabla^{2} \vec{V}^{\prime}=\frac{1}{2} M a_{\lambda}^{1 / 2} \lambda^{1 / 2} \Phi \nabla T^{*}$,

$\nabla^{2} \Phi=\frac{1}{2} M a_{\lambda}^{1 / 2} \lambda^{1 / 2} \vec{V}^{\prime} \nabla T^{*}$

The boundary conditions at the center of the droplet are

$\vec{V}^{\prime}=0, \quad \Phi=$ finite.

On the free surface, one has

$$
\begin{aligned}
& M a_{\lambda}^{1 / 2} \lambda^{1 / 2} u_{R}^{\prime}=\frac{\partial E v}{\partial T^{*}} \Phi \\
& R \frac{\partial}{\partial R}\left(\frac{u_{\theta}^{\prime}}{R}\right)+\frac{1}{R} \frac{\partial u_{R}^{\prime}}{\partial \theta}=-\frac{M a_{\lambda}^{1 / 2} \lambda^{-1 / 2}}{2 R} \frac{\partial \Phi}{\partial \theta} \\
& \frac{1}{R \sin \theta} \frac{\partial u_{R}^{\prime}}{\partial \phi}+R \frac{\partial}{\partial R}\left(\frac{u_{\phi}^{\prime}}{R}\right)=-\frac{M a_{\lambda}^{1 / 2} \lambda^{-1 / 2}}{2 R \sin \theta} \frac{\partial \Phi}{\partial \phi} \\
& -\Pi+2 \operatorname{Pr} \frac{\partial u_{R}^{\prime}}{\partial R}=\operatorname{Pr} M a_{\lambda}^{1 / 2} \lambda^{-1 / 2} \frac{\Phi}{R} \\
& -\frac{\partial \Phi}{\partial R}=\chi \Phi+\frac{1}{2} M a_{\lambda}^{1 / 2} \lambda^{-1 / 2} \frac{\partial u_{R}^{\prime}}{\partial \phi}
\end{aligned}
$$


Moreover, at $\theta=\pi$ and 0 , it must satisfy

$\left[\frac{\partial\left(\sin \theta u_{\phi}^{\prime}\right)}{\partial \theta}-\frac{\partial u_{\theta}^{\prime}}{\partial \phi}\right]_{\theta=\pi}-\left[\frac{\partial\left(\sin \theta u_{\phi}^{\prime}\right)}{\partial \theta}-\frac{\partial u_{\theta}^{\prime}}{\partial \phi}\right]_{\theta=0}=0$.

The maximum of eigenvalues $1 / M a_{\lambda}$ coincides with the solution of system (3.6). Hence, it follows that

$\widetilde{M} a_{\mathrm{c}}=\min _{\mathscr{T}} M a_{\lambda}$

for any positive set of the eigenvalues $M a_{\lambda}$ in $\mathscr{T}$ which is identical with $\mathscr{H}$ except for the removal of the continuity condition on $\vec{V}^{\prime}$. Then the eigenvalue problem and the optimum stability boundary are related by

$M a_{\mathrm{c}}=\max _{\lambda>0} \widetilde{M a_{\mathrm{c}}}=\max _{\lambda>0}\left(\min _{\mathscr{T}} M a_{\lambda}\right)$.

Eqs. (2.38), (3.9) and (3.10) are linear with time dependence through $T^{*}$ and without limitation on the magnitude of the disturbances. This fact eliminates the necessity of making an arbitrary decision regarding the onset of instability based on disturbance amplification or its growth rate.

\subsection{Solution procedure}

By requiring the disturbance energy to decrease exponentially, the non-linear governing equations of the perturbed field, Eqs. (2.38)-(2.40), are reformulated to a maximum problem (3.6). The best estimation of the limits of stability, i.e., the optimum stability boundary, is then obtained by finding the minimum eigenvalue of the Euler-Lagrange equations associated with the maximum problem. Their relation is shown in Eq. (3.18). In the following, the eigenvalue problem, Eqs. (2.38), (2.41), (2.42) and (3.9)-(3.16), is solved numerically. The results are then discussed and compared with those obtained by the linear stability analysis [19,20].

By virtue of the continuity equation, the two tangential stress boundary conditions, Eqs. (3.12) and (3.13), can be combined into a single one:

$$
\frac{\partial^{2}}{\partial R^{2}}\left(R u_{R}^{\prime}\right)-\frac{\left(2+L^{2}\right)\left(R u_{R}^{\prime}\right)}{R^{2}}=\frac{M a_{\lambda}^{1 / 2} \lambda^{-1 / 2}}{2 R} L^{2} \Phi,
$$

where

$$
\begin{aligned}
L^{2} & =\frac{1}{\sin \theta} \frac{\partial}{\partial \theta}\left(\sin \theta \frac{\partial}{\partial \theta}\right)+\frac{1}{\sin ^{2} \theta} \frac{\partial^{2}}{\partial \phi^{2}}, \\
& =R^{2} \nabla^{2}-\frac{\partial}{\partial R}\left(R^{2} \frac{\partial}{\partial R}\right)
\end{aligned}
$$

with $\nabla^{2}$ denoting the Laplace operator in the spherical coordinates, i.e.,

$$
\begin{aligned}
\nabla^{2}= & \frac{1}{R^{2}} \frac{\partial}{\partial R}\left(R^{2} \frac{\partial}{\partial R}\right)+\frac{1}{R^{2} \sin \theta} \frac{\partial}{\partial \theta}\left(\sin \theta \frac{\partial}{\partial \theta}\right) \\
& +\frac{1}{R^{2} \sin ^{2} \theta} \frac{\partial^{2}}{\partial \phi^{2}} .
\end{aligned}
$$

By eliminating the $\Pi$-term in Eq. (3.9), the perturbed $R$-momentum equation in terms of $u_{R}^{\prime}$ can be derived as follows:

$\nabla^{4}\left(R u_{R}^{\prime}\right)-\frac{1}{2 R} M a_{\lambda}^{1 / 2} \lambda^{1 / 2} L^{2}\left(\Phi \frac{\partial T^{*}}{\partial R}\right)=0$.

Motivated by the operator $L^{2}$, the disturbed quantities are expressed in terms of the spherical harmonics, i.e.,

$R u_{R}^{\prime}=U(R) Y_{1}^{m}(\theta, \phi)$,

$\Phi=\mathfrak{J}(R) Y_{1}^{m}(\theta, \phi)$.

The spherical harmonics $Y_{1}^{m}$ satisfies the following equation

$$
\begin{aligned}
& {\left[\frac{1}{\sin \theta} \frac{\partial}{\partial \theta}\left(\sin \theta \frac{\partial}{\partial \theta}\right)+\frac{1}{\sin ^{2} \theta} \frac{\partial^{2}}{\partial \phi^{2}}\right] Y_{1}^{m}(\theta, \phi)} \\
& \quad=-l(l+1) Y_{1}^{m}(\theta, \phi),
\end{aligned}
$$

wherein

$Y_{1}^{m}(\theta, \phi)=P_{1}^{m}(\cos \theta) e^{\mathrm{i} m \phi}$,

with $P_{1}^{m}(\cos \theta)$ being the associated Legendre polynomials.

Substitution of Eq. (3.23), (3.24) into Eqs. (3.10) and (3.22), and the boundary conditions (2.41), (2.42), (3.11), (3.15), (3.16), and (3.19) gives

$$
\begin{aligned}
& {\left[\frac{\mathrm{d}^{2}}{\mathrm{~d} R^{2}}+\frac{2}{R} \frac{\mathrm{d}}{\mathrm{d} R}-\frac{l(l+1)}{R^{2}}\right]\left[\frac{\mathrm{d}^{2}}{\mathrm{~d} R^{2}}+\frac{2}{R} \frac{\mathrm{d}}{\mathrm{d} R}-\frac{l(l+1)}{R^{2}}\right] U} \\
& =-\frac{1}{2} M a_{\lambda}^{1 / 2} \lambda^{1 / 2} \frac{l(l+1)}{R} \frac{\partial T^{*}}{\partial R} \mathfrak{J} \\
& {\left[\frac{\mathrm{d}^{2}}{\mathrm{~d} R^{2}}+\frac{2}{R} \frac{\mathrm{d}}{\mathrm{d} R}-\frac{l(l+1)}{R^{2}}\right] \mathfrak{J}} \\
& =\frac{1}{2} M a_{\lambda}^{1 / 2} \lambda^{1 / 2} \frac{U}{R} \frac{\partial T^{*}}{\partial R}
\end{aligned}
$$

at $R=0$,

$U=0, \quad \mathfrak{I}=$ finite,

at $R=1$,

$$
\begin{aligned}
& M a_{\lambda}^{1 / 2} \lambda^{1 / 2} \frac{U}{R}=\frac{\partial E v}{\partial T^{*}} \mathfrak{I}, \\
& \frac{\mathrm{d}^{2} U}{\mathrm{~d} R^{2}}-\frac{2-l(l+1)}{R^{2}} U=-\frac{1}{2} M a_{\lambda}^{1 / 2} \lambda^{-\frac{1}{2}} \frac{l(l+1)}{R} \mathfrak{J}, \\
& -\frac{\mathrm{d} \mathfrak{I}}{\mathrm{d} R}=\chi \mathfrak{I}+\frac{1}{2} M a_{\lambda}^{1 / 2} \lambda^{-\frac{1}{2}}\left(\frac{1}{R} \frac{\mathrm{d} U}{\mathrm{~d} R}-\frac{U}{R^{2}}\right) .
\end{aligned}
$$


The determination of $M a_{\mathrm{c}}$ at a fixed time is now accomplished by maximizing the least positive eigenvalue $M a_{\lambda}$ of Eqs. (3.27) and (3.28) with boundary conditions (3.29)-(3.33) over the free parameter $\lambda$, and then minimizing them over the domain of wave number $l$, i.e.

$M a_{\mathrm{c}}=\min _{1}\left[\max _{\lambda}\left(\min _{\mathscr{T}} M a_{\lambda}\right)\right]$.

Eigenvalues are computed using a FORTRAN program with the IMSL subroutine DGVLRG and accurate to the fourteenth decimal with the double-precision arithmetic on PC. Using a non-uniform grid of 1000 points, the eigenvalue problem is solved for each specified time instant and initial temperature with various values of $\lambda$, and for $l$ between 1 and 10 .

\subsection{Results and discussion}

The stability conditions are calculated, as an example, for an octane droplet at various time instants and initial temperatures. However, the present method can also be applied to other droplets, e.g., heptane, at their early stage of evaporation and with an initial temperature not close to the boiling point. The values of the physical properties of octane are referred to the book edited by Dauber and Danner [43].

Fig. 2 are the stability curves calculated for an octane droplet at various time instants and two initial temperatures, $T_{0}=293$ and $353 \mathrm{~K}$. An experimental trajectory would be represented by a horizontal line on this plot. The droplet remains stable until the instant at which the

(a)

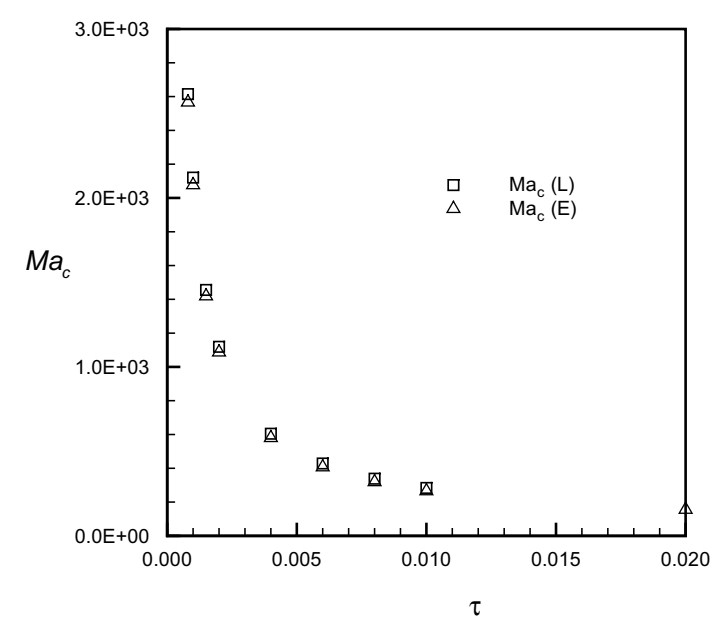

stability boundary is crossed. Hence, the stability curves shown in Fig. 2 represent the lower bound of the onset time of instability of an octane droplet. It can be seen from this figure that, with a smaller value of the initial Marangoni number, which indicates a smaller driving potential for instability, the droplet remains stable for a longer period of time. Moreover, this stable time period increases rapidly for the initial Marangoni number less than about 400. That means when the initial Marangoni number is less than 400 , the Marangoni effect is not large enough to trigger the instability easily. Fig. 2 also indicates that as time proceeds, because of the surface temperature reduction together with the consequent variation in surface tension due to disturbances, the droplet tends to be more unstable.

Fig. 3 gives the onset Marangoni number as a function of wave numbers at different time instants. Calculations for four initial temperatures are included for comparison. A lot of information can be learned from this figure. (1) Each curve in Fig. 3 represents the variation of the onset Marangoni number versus wave number. The minimum point on each curve denotes the critical point. The corresponding onset Marangoni number and wave number are called critical Marangoni number, $M a_{\mathrm{c}}$, and critical wave number, $l_{\mathrm{c}}$. (2) The critical wave number increases with the increase of the initial temperature. That means disturbances of shorter wave lengths, i.e., larger wave numbers, can become unstable only at a higher initial temperature. (3) As time proceeds, the critical Marangoni number decreases indicating that the evaporating droplet becomes more unstable, as shown in Fig. 2. In the mean time, the critical wave number shifts back to a lower value in

(b)

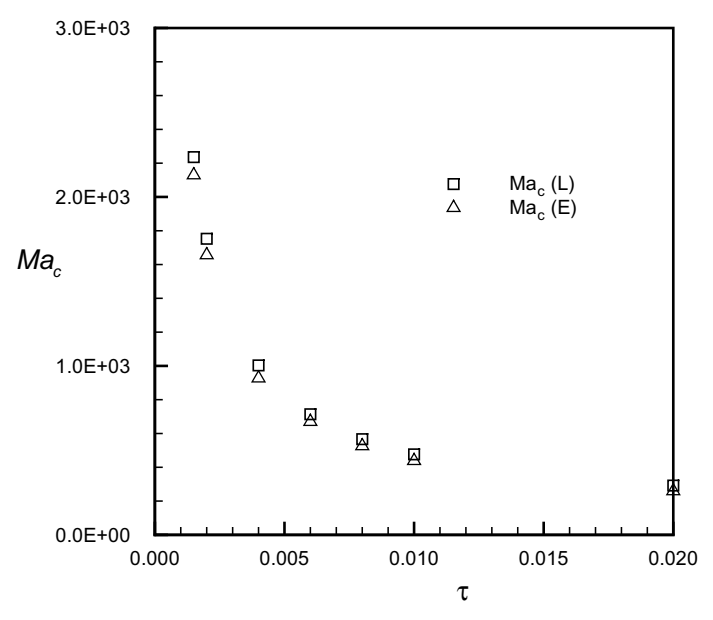

Fig. 2. Stability curves as a function of time for an octane droplet with a non-deformable free surface at two different initial temperatures: (a) $T_{0}=293 \mathrm{~K}$, (b) $T_{0}=353 \mathrm{~K}$; (E) energy method, (L) linear stability analysis. 
(a)

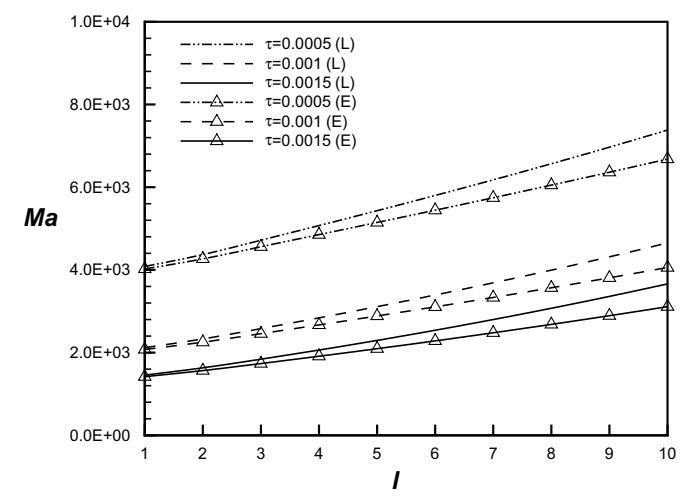

(c)

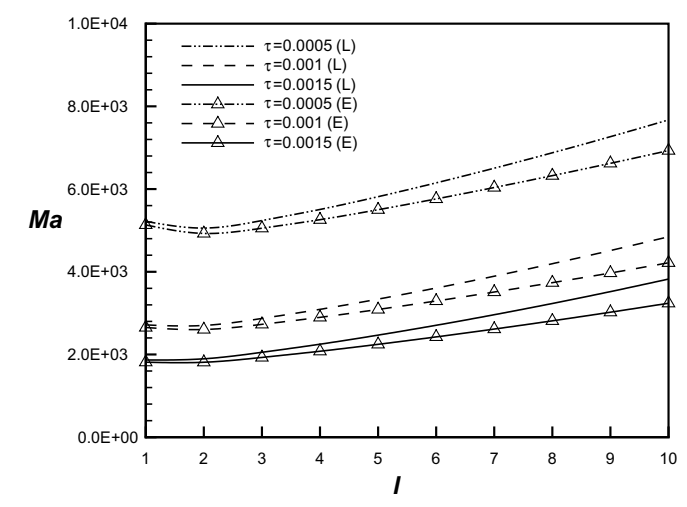

(b)

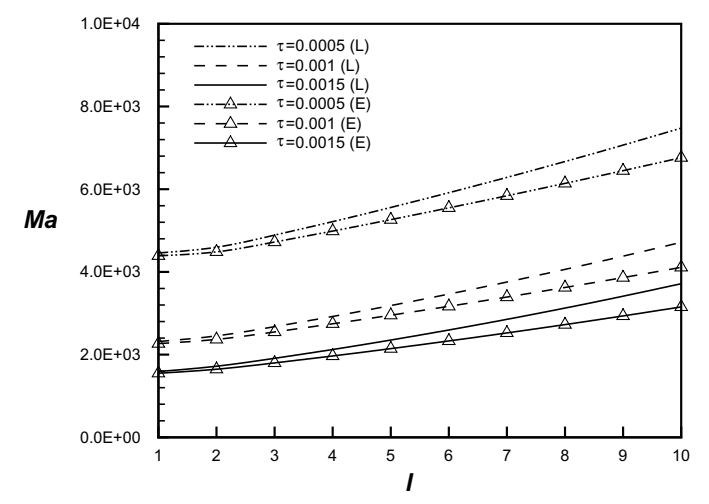

(d)

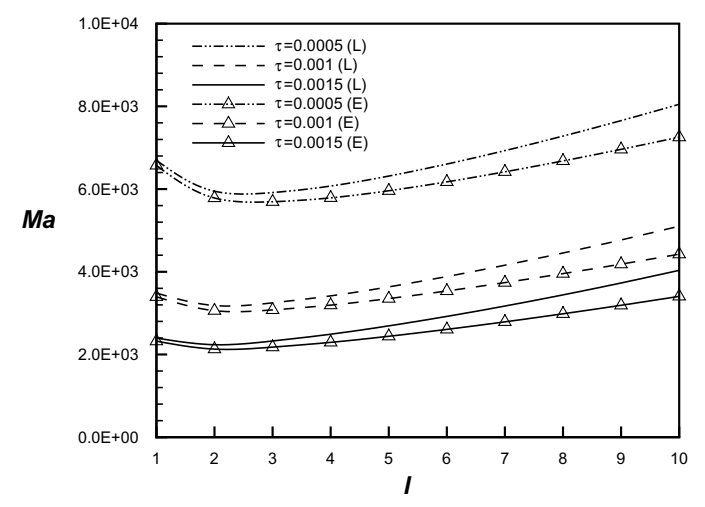

Fig. 3. Stability curves as a function of wave number for an octane droplet with a non-deformable free surface at various instants: (a) $T_{0}=293 \mathrm{~K}$, (b) $T_{0}=313 \mathrm{~K}$, (c) $T_{0}=333 \mathrm{~K}$, (d) $T_{0}=353 \mathrm{~K}$; (E) energy method, (L) linear stability analysis.

accord with the growth of the thermal boundary layer thickness near the free surface. To illustrate this phenomenon more clearly, the numerically calculated values of $M a_{\mathrm{c}}$ and $l_{\mathrm{c}}$ for an octane droplet at different initial temperatures and specified time instants are tabulated in Table 1.

Shown in Fig. 4 is the stability curves as a function of the droplet initial temperature, $T_{0}$. It is obvious that the onset Marangoni number increases with $T_{0}$. That means, with a higher initial temperature, the droplet seems to possess intrinsically a certain potential to damp the disturbances and, as a result, is more stable. The mechanism is not obvious simply from the numerically calculated results. However, the analytic expression of $M a_{\mathrm{c}}$ in terms of the temperature distribution as derived from the linear stability analysis $[19,20]$ could provide a clear and direct understanding of the initial-temperature effect on the onset of Marangoni instability of an evaporating droplet. According to the explanation by $\mathrm{Ha}$ and Lai [20], there exist two competing mechanisms determining the onset of instability. One of them is the temperature reduction near the free surface which is the driving potential for the Marangoni instability. Therefore, with a higher initial temperature which results in a larger temperature reduction near the free surface, the droplet on one hand should become more unstable. The other mechanism is the existence of heat transfer to the surrounding gas due to evaporation, which tends to smooth the surface temperature disturbances and increases with initial temperature. Thus, the droplet, on the other hand, becomes more stable with a higher initial temperature. The competition between these two mechanisms determines the effect of the initial temperature on the droplet instability and finally leads to the result that $M a_{\mathrm{c}}$ increases with $T_{0}$.

In order to further support the validity of the above explanation, the results obtained from the linear stability analysis with a quasi-steady approximation $[19,20]$ are also shown in Figs. 2-4 and Table 1. It is obvious by comparison that the energy method and the linear stability analysis predict similar trends for the onset of Marangoni instability of an evaporating droplet. The 
Table 1

Numerically calculated values of $M a_{\mathrm{c}}$ and $l_{\mathrm{c}}$ for an octane droplet at different time instants with initial temperature $T_{0}=293$ and $353 \mathrm{~K}$

\begin{tabular}{|c|c|c|c|c|c|}
\hline \multirow[b]{2}{*}{$T_{0}$} & \multirow[b]{2}{*}{$\tau$} & \multicolumn{2}{|c|}{ Linear stability analysis } & \multicolumn{2}{|c|}{ Energy method } \\
\hline & & $M a_{\mathrm{c}}$ & $l_{\mathrm{c}}$ & $M a_{\mathrm{c}}$ & $l_{\mathrm{c}}$ \\
\hline 293 & 0.0004 & $5.06 \times 10^{3}$ & 1 & $4.99 \times 10^{3}$ & 1 \\
\hline 293 & 0.0005 & $4.08 \times 10^{3}$ & 1 & $4.02 \times 10^{3}$ & 1 \\
\hline 293 & 0.0006 & $3.43 \times 10^{3}$ & 1 & $3.38 \times 10^{3}$ & 1 \\
\hline 293 & 0.0008 & $2.61 \times 10^{3}$ & 1 & $2.57 \times 10^{3}$ & 1 \\
\hline 293 & 0.001 & $2.12 \times 10^{3}$ & 1 & $2.08 \times 10^{3}$ & 1 \\
\hline 293 & 0.0015 & $1.46 \times 10^{3}$ & 1 & $1.42 \times 10^{3}$ & 1 \\
\hline 293 & 0.002 & $1.12 \times 10^{3}$ & 1 & $1.09 \times 10^{3}$ & 1 \\
\hline 353 & 0.0005 & $5.92 \times 10^{3}$ & 3 & $5.69 \times 10^{3}$ & 3 \\
\hline 353 & 0.001 & $3.18 \times 10^{3}$ & 2 & $3.06 \times 10^{3}$ & 2 \\
\hline 353 & 0.0015 & $2.24 \times 10^{3}$ & 2 & $2.13 \times 10^{3}$ & 2 \\
\hline 353 & 0.002 & $1.75 \times 10^{3}$ & 2 & $1.66 \times 10^{3}$ & 2 \\
\hline 353 & 0.004 & $1.00 \times 10^{3}$ & 1 & $9.27 \times 10^{2}$ & 2 \\
\hline 353 & 0.006 & $7.14 \times 10^{2}$ & 1 & $6.70 \times 10^{2}$ & 1 \\
\hline 353 & 0.008 & $5.66 \times 10^{2}$ & 1 & $5.26 \times 10^{2}$ & 1 \\
\hline 353 & 0.01 & $4.76 \times 10^{2}$ & 1 & $4.39 \times 10^{2}$ & 1 \\
\hline 353 & 0.02 & $2.92 \times 10^{2}$ & 1 & $2.60 \times 10^{2}$ & 1 \\
\hline
\end{tabular}

(a) $I=1$

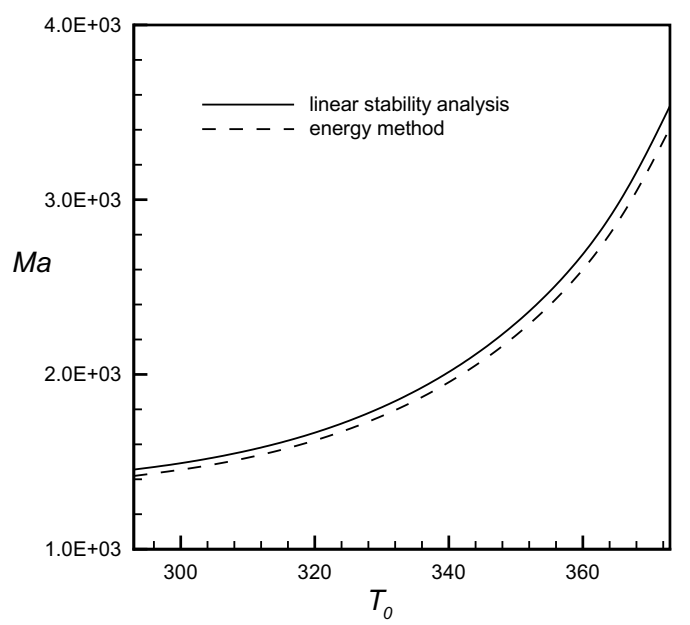

(b) $I=5$

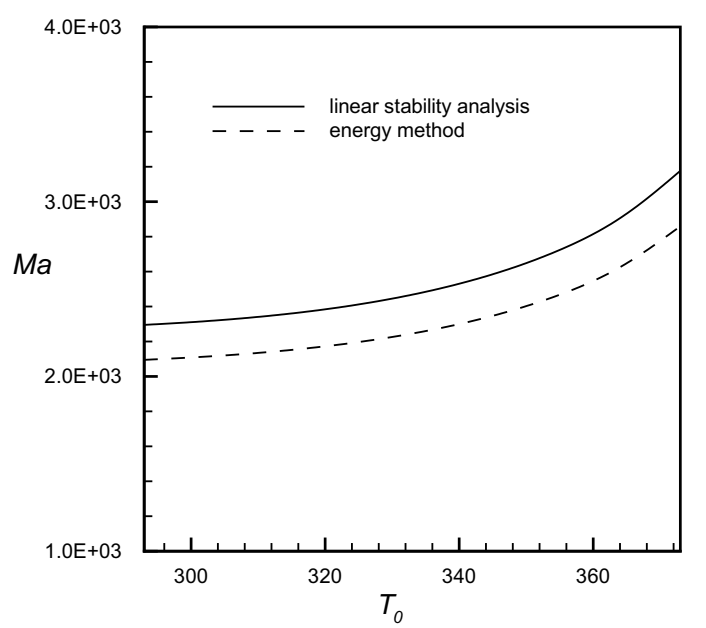

Fig. 4. Stability curves as a function of initial temperature for an octane droplet with a non-deformable free surface at $\tau=0.0015$ for $l=1$ and 5; (E) energy method, (L) linear stability analysis.

critical Marangoni number, $M a_{\mathrm{c}}$, predicted by the linear stability analysis is a little higher than that predicted by the energy method. The subcritical region between the $M a_{\mathrm{c}}$ 's as calculated by these two methods is very narrow. This fact demonstrates that although the linear stability analysis with a quasi-steady approximation is not theoretically and rigorously accurate for a time-varying flow motion, it actually provides a pretty good approximation, at least for the present situation. Therefore, the basic physics as demonstrated by the linear stability analysis should be very meaningful.

\section{Conclusions and suggestions}

The onset of Marangoni instability of a motionless evaporating droplet surrounded by a passive gas is studied theoretically by the energy method. The results clearly demonstrate the validity of energy method as applied to flows whose basic states vary with time. Unlike linear stability analysis, energy method is applicable to flows that are not necessarily slowly varying. Moreover, energy method provides an ascertainment of stability against disturbances of any amplitude. The present 
results also indicate that, as time proceeds, both the increase of surface temperature reduction and the growth of the thermal boundary layer near the free surface are conducive to the onset of instability. The critical Marangoni number and wave number increase with the droplet initial temperature. In addition, the results as predicted by linear stability analysis $[19,20]$ are in accord with the present results, indicating the validity of linear stability analysis as a first approximation to the analysis of Marangoni instability of an evaporating droplet.

However, during the evaporation process, the position of the free surface is subject to a certain change due to the boundary regression. Although the time scale of the interface regression is, in general, about one order larger than that of the heat diffusion in the liquid phase, the effect of boundary regression may become significant to the flow instability for a highly volatile liquid or when the initial temperature is close enough to its boiling point. A modified model is then needed to incorporate appropriately the surface regression effect. Moreover, to understand the evolution of disturbances when coupled with a time-varying flow field is also very important and interesting. To do so, a stochastic formulation with a statistically random distribution of the initial disturbances is needed. The onset time of instability for any specified Marangoni number can then be determined and compared with the experimental observation.

\section{Acknowledgements}

The support of the ROC National Science Council through Grant NSC89-2212-E002-063 is gratefully acknowledged.

\section{References}

[1] J.R.A. Pearson, On convection cells induced by surface tension, J. Fluid Mech. 4 (1958) 489-500.

[2] O. Reynolds, On the dynamical theory of incompressible viscous fluids and the determination of the criterion, Philos. Trans. Roy. Soc. Lond. A 186 (1895) 123-164.

[3] W.McF. Orr, The stability or instability of the steady motions of a liquid. Part II: A viscous liquid, Proc. Roy. Irish Acad. A 27 (1907) 69-138.

[4] J. Serrin, On the stability of viscous fluid motions, Arch. Ration. Mech. Anal. 3 (1959) 1-13.

[5] D.D. Joseph, On the stability of the Boussinesq equations, Arch. Ration. Mech. Anal. 20 (1965) 59-71.

[6] D.D. Joseph, Nonlinear stability of the Boussinesq equations by the method of energy, Arch. Ration. Mech. Anal. 22 (1966) 163-184.

[7] G.M. Homsy, Global stability of time-dependent flows: impulsively heated or cooled fluid layers, J. Fluid Mech. 60 (1973) 129-139.
[8] G.P. Neitzel, Onset of convection in impulsively heated or cooled fluid layers, Phys. Fluids 25 (1982) 210-211.

[9] S.H. Davis, C. Von Kerczek, A reformulation of energy stability theory, Arch. Ration. Mech. Anal. 52 (1973) 117122.

[10] G.M. Homsy, Global stability of time-dependent flows. Part 2. Modulated fluid layers, J. Fluid Mech. 62 (1974) 387-403.

[11] R.J. Gumerman, G.M. Homsy, The stability of uniformly accelerated flows with application to convection driven by surface tension, J. Fluid Mech. 68 (1975) 191-207.

[12] S.H. Davis, G.M. Homsy, Energy stability theory for freesurface problems: buoyancy-thermocapillary layers, J. Fluid Mech. 98 (1980) 527-553.

[13] G.P. Neitzel, S.H. Davis, Energy stability theory of decelerating swirl flows, Phys. Fluids 23 (1980) 432-437.

[14] G.P. Neitzel, Marginal stability of impulsively initial Couette flow and spin-decay, Phys. Fluids 25 (1982) 226231.

[15] G.P. Neitzel, Stability of circular Couette flow with variable inner cylinder speed, J. Fluid Mech. 123 (1982) 43-57.

[16] R.D. Benguria, M.C. Depassier, Oscillatory instabilities in the Rayleigh-Benard problem with a free surface, Phys. Fluids 30 (1987) 1678-1682.

[17] R.D. Benguria, M.C. Depassier, On the linear stability theory of Benard-Marangoni convection, Phys. Fluids A 1 (1989) 1123-1127.

[18] S.H. Davis, Buoyancy-surface tension instability by the method of energy, J. Fluid Mech. 39 (1969) 347-359.

[19] V.M. Ha, The analysis of Marangoni instability of evaporating liquid, Ph.D. Dissertation, Department of Mechanical Engineering, National Taiwan University, 1998.

[20] V.M. Ha, C.L. Lai, The onset of stationary Marangoni instability of an evaporating droplet, Proc. Roy. Soc. Lond. Ser. A 457 (2001) 885-909.

[21] C.L. McTaggart, On the stabilizing effect of surface films in Benard convection, PCH. Physicochem. Hydrodynam. 5 (1984) 321-331.

[22] C. Perez-Garcia, G. Carneiro, Linear stability analysis of Benard-Marangoni convection in fluids with a deformable free surface, Phys. Fluids A 3 (1991) 292-298.

[23] Y. Renardy, M. Renardy, Perturbation analysis of steady and oscillatory onset in a Benard problem with two similar liquids, Phys. Fluids 28 (1985) 2699-2708.

[24] Y. Renardy, Interfacial stability in a two-layer Benard problem, Phys. Fluids 29 (1986) 358-363.

[25] K.A. Smith, On convective instability induced by surfacetension gradients, J. Fluid Mech. 24 (1966) 401-414.

[26] C.V. Sternling, L.E. Scriven, Interfacial turbulence: hydrodynamic instability and the Marangoni effect, Am. Inst. Chem. Engin. J. 5 (1959) 514-523.

[27] M. Takashima, Nature of the neutral state in convective instability induced by surface tension and buoyancy, J. Phys. Soc. Jpn. 28 (1970) 810.

[28] M. Takashima, Surface tension driven instability in a horizontal liquid layer with a deformable free surface. II. Overstability, J. Phys. Soc. Jpn. 50 (1981) 2715-2756.

[29] S. Wahal, A. Bose, Rayleigh-Benard and interfacial instabilities in two immiscible liquid layers, Phys. Fluids 31 (1988) 3502-3510. 
[30] R.W. Zeren, W.C. Reynolds, Thermal instabilities in twofluid horizontal layers, J. Fluid Mech. 53 (1972) 305-327.

[31] T.S. Sorensen, M. Hennenberg, A. Steinchen, A. Sanfeld, Chemical and hydrodynamical analysis of stability of a spherical interface, J. Colloid Interface Sci. 56 (1975) 191205.

[32] F.J. Higuera, A. Linan, Stability of a droplet vaporizing in a hot atmosphere, Progr. Astronaut. Aeronaut. 105 (1985) 217-238.

[33] O. Pirotte, G. Lebon, Surface-tension driven instability in spherical shells, Appl. Micrograv. Technol. 1 (1988) 175179.

[34] A. Cloot, G. Lebon, Marangoni convection in a rotating spherical geometry, Phys. Fluids A 2 (1990) 525-529.

[35] A. Cloot, G. Lebon, Surface deformation effect on Marangoni convection in a spherical shell, Micrograv. Sci. Technol. 3 (1990) 44-46.

[36] H.C.J. Hoefsloot, H.W. Hoogstraten, Marangoni instability in spherical shells, Appl. Micrograv. Technol. 2 (1989) $106-108$.

[37] H.C.J. Hoefsloot, H.W. Hoogstraten, L.P.B.M. Janssen, Marangoni instability in a liquid layer confined between two concentric spherical surfaces under zero-gravity conditions, Appl. Sci. Res. 47 (1990) 357-377.

[38] H.C.J. Hoefsloot, H.W. Hoogstraten, L.P.B.M. Janssen, J.W. Knobbe, Growth factors for Marangoni instability in a spherical liquid layer under zero-gravity conditions, Appl. Sci. Res. 49 (1992) 161-173.

[39] A.-T. Chai, N. Rashidnia, V.S. Arpaci, Marangoni instability induced convection in an evaporating liquid droplet, Proceedings VIII European Symposium on Materials and Fluid Sciences in Microgravity, Brussels, Belgium, 1992, pp. 187-192.

[40] G. Lebon, P.C. Dauby, A. Cloot, Some problem raised by Marangoni instability in spherical geometry, in: H.J. Rath (Ed.), Microgravity Fluid Dynamic, Springer, 1992, pp. 71-79.

[41] S.K. Wilson, The onset of steady Marangoni convection in a spherical geometry, J. Eng. Math. 28 (1994) 427445.

[42] K.A. Hoffmann, Computational Fluid Dynamics for Engineers, EES, 1989, p. 255.

[43] T.E. Dauber, R.P. Danner, Data compilation tables of properties of pure compounds, New York, 1985. 